\title{
A 39-Year-Old Male Congenital Tricuspid Atresia Patient Who Presented with a New Axillary Lesion after an Orthotopic Heart Transplant
}

\author{
Zizi Yu ${ }^{a}$ Nicholas Theodosakis ${ }^{b}$ Megan J. Fitzpatrick ${ }^{c}$ Ruth K. Foreman ${ }^{c}$ \\ Bonnie Mackool ${ }^{\mathrm{b}}$ \\ ${ }^{a}$ Harvard Medical School, Boston, MA, USA; ${ }^{b}$ Department of Dermatology, Massachusetts \\ General Hospital, Boston, MA, USA; ' Department of Pathology, Massachusetts General \\ Hospital, Boston, MA, USA
}

\section{Keywords}

Mucormycosis · Fungal infection · Immunosuppression

\section{Abstract}

Mucormycosis is a rare and aggressive fungal infection, most often caused by species of the Mucor, Rhizomucor, Rhizopus, Absidia, and Cunninghamella genera. The condition most commonly affects patients with uncontrolled diabetes, HIV/AIDS, malignancy, and those receiving long-term immunosuppressive therapy. We report the case of a 39-year-old male with biopsy-proven cutaneous mucormycosis of the left axilla 4 months after an orthotopic heart transplant for congenital tricuspid atresia.

\section{Case Report}

We were asked to evaluate a 39-year-old hospitalized male with a history of congenital tricuspid atresia, who had had a left axillary wound for 1 week in the absence of trauma. Four months previously, he had undergone an orthotopic heart transplant with a postoperative course complicated by hemorrhagic shock and resultant renal failure and cirrhosis. His medications included prednisone, mycophenolate mofetil, cyclosporin, micafungin, and fluconazole. He received the last 2 of these medications for infectious complications of immobility 
Fig. 1. A 3-cm superficial lesion, with a central $0.6-\mathrm{cm}$ black eschar and a rim of fibrinous tissue in the anterior left axilla. Two 3-mm punch biopsies were obtained from the edge of the eschar for $\mathrm{H} \& \mathrm{E}$ and tissue culture.

Yu et al.: Congenital Tricuspid Atresia with a New Axillary Lesion after an Orthotopic Heart Transplant

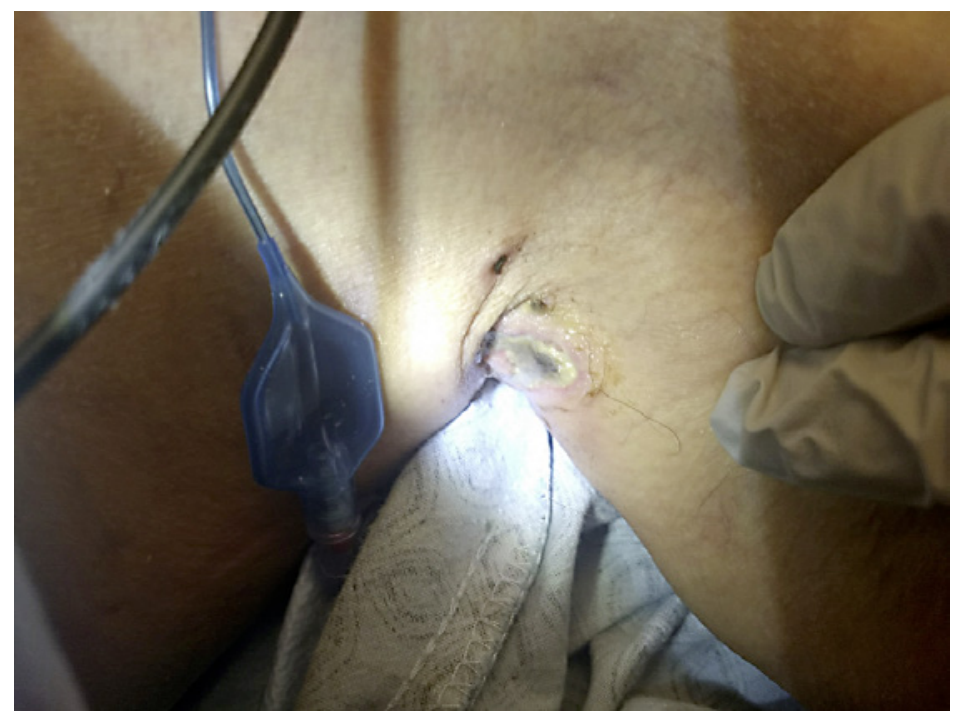

and immunosuppression, including multiple decubitus ulcers, sacral osteomyelitis, and Candida parapsilosis peritonitis and fungemia. Examination revealed a lesion $3 \mathrm{~cm}$ in diameter with a roughened granulating surface in the left axillary vault (Fig. 1). A dark hemorrhagic crust was present centrally and measured $0.6 \mathrm{~cm}$ in diameter. The lesion was bordered by erythema and was tender to palpation. We performed a skin biopsy for histology, tissue wet prep, and culture.

\section{Diagnosis and Clinical Course}

Histopathologic examination, tissue culture, and fungal wet prep revealed thick, nonseptate hyphae consistent with Mucorales infection (Fig. 2, 3). The hyphae stained positively on Periodic acid-Schiff/diastase stain. Significant necrosis, with marked neutrophilic infiltrate in the overlying epidermis and dermis, was also seen. Fungal culture revealed deep tissue infection with Rhizopus and C. parapsilosis. Amphotericin B liposomal formulation ( $5 \mathrm{mg} / \mathrm{kg}$ i.v.) was initiated as an antifungal therapy.

Chest CT revealed subcutaneous inflammation and axillary lymphadenopathy without obvious invasion of the chest wall. The patient underwent operative debridement and 2 subsequent bedside debridements prior to obtaining clear margins. Posaconazole $(300 \mathrm{mg}$ i.v. $2 \times$ daily) was added to his antifungal regimen. Cyclosporine was reduced to an oral dose of $25 \mathrm{mg} 2 \times$ daily due to the risk of an adverse drug interaction. Posaconazole was discontinued 1 month later due to elevated values on liver function testing. Liposomal amphotericin B was continued for another month, until his death due to recurrent idiopathic status epilepticus.

\section{Discussion}

Mucormycosis is a rare and aggressive fungal infection, most frequently caused by the Rhizopus and Mucor genera of Mucorales fungi [1]. Mucorales is ubiquitous worldwide but is implicated in a minority of fungal infections, i.e., $8-13 \%$ as observed on autopsies of high-risk patients [1]. Risk factors for mucormycosis include poorly controlled diabetes mellitus 


\begin{tabular}{l|l}
\hline DOI: 10.1159/000503661 & $\begin{array}{l}\text { (c) } 2019 \text { The Author(s). Published by S. Karger AG, Basel } \\
\text { www.karger.com/dpa }\end{array}$ \\
\hline Yu et al.: Congenital Tricuspid Atresia with a New Axillary Lesion after an Orthotopic
\end{tabular}

Yu et al.: Congenital Tricuspid Atresia with a New Axillary Lesion after an Orthotopic Heart Transplant

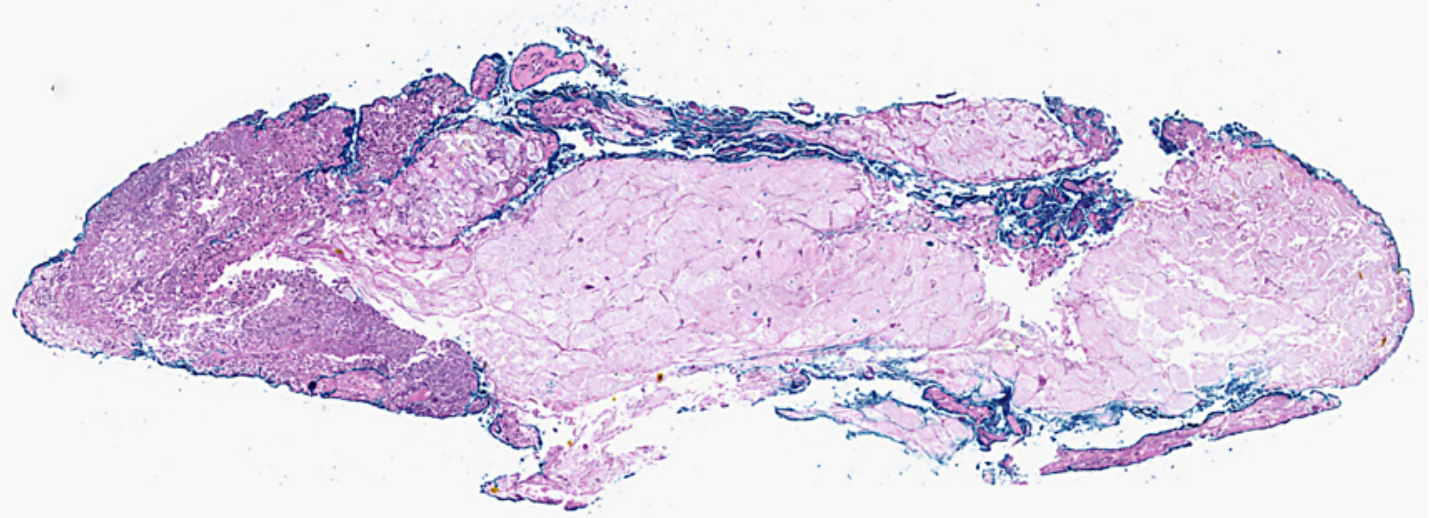

a

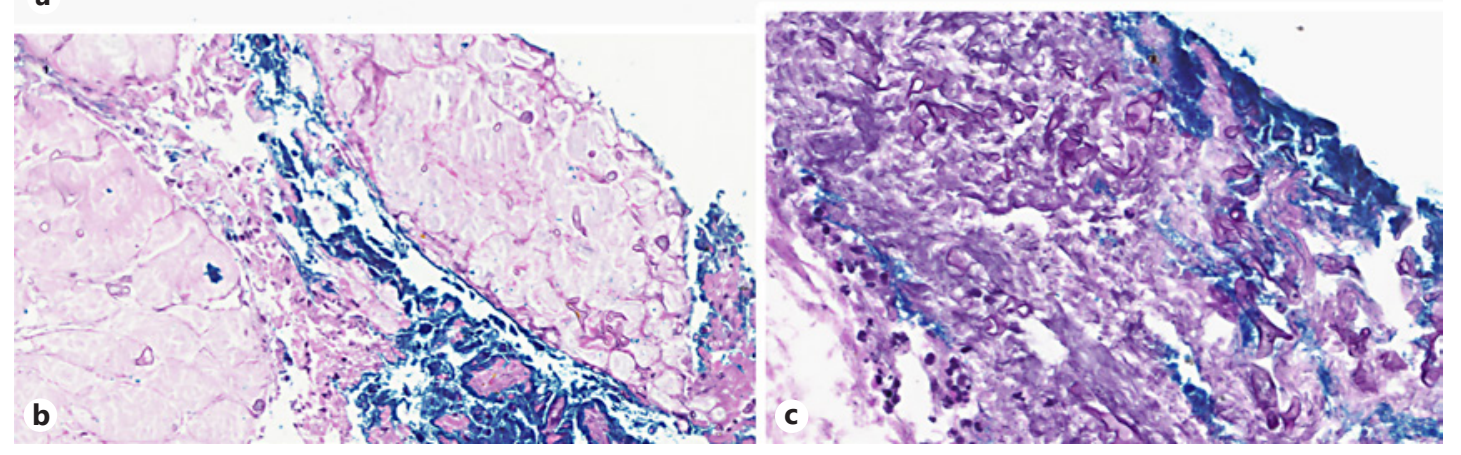

Fig. 2. Punch biopsy of the left axillary lesion. a Low-power view. H\&E. b Higher-power view demonstrating broad, aseptate, branching fungal hyphae. H\&E. c Positive staining of fungal hyphae. PAS/D.
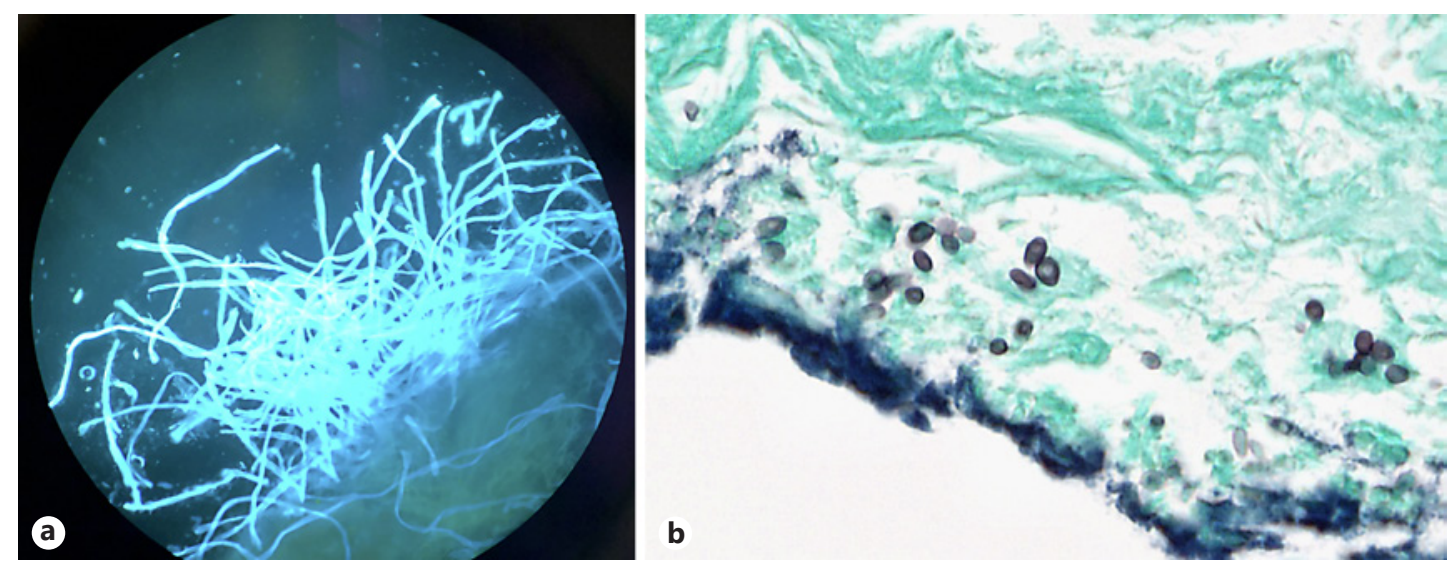

Fig. 3. a Wet prep showing broad, ribbon-like hyphae. b A second population of yeast forms, consistent with C. parapsilosis. Grocott-Gomori methenamine silver (GMS).

(particularly diabetic ketoacidosis), HIV/AIDS, solid organ transplantation, malignancy, hematological disease with or without hematopoietic stem cell transplantation, iron overload, malnutrition, and prolonged glucocorticoid and/or other immunosuppressive therapy [1].

Cutaneous mucormycosis is the third most common presentation of the disease after rhinocerebral and pulmonary mucormycosis. Mucorales can spread via the inhalation or 
ingestion of spores, or by direct inoculation in immunocompromised hosts [2]. Patients with disruptions in the normal skin barrier due to burns, traumatic disruption, catheter insertion, injections, or persistent maceration of skin, are at an increased risk of developing cutaneous mucormycosis [3]. The disease can often invade locally and penetrate into adjacent fat, muscle, fascia, and even bone, while secondary vascular invasion and hematogenous spread to the deep organs are less common $[1,4]$. Isolated cutaneous mucormycosis without dissemination is associated with a more favorable prognosis and lower mortality when diagnosed and treated promptly [3].

Determining the infectious cause of a cutaneous lesion in a severely immunocompromised patient, particularly one with a complex history of polymicrobial infections, typically requires biopsy for both $\mathrm{H} \& \mathrm{E}$ and culture. Biopsy of the axillary lesion showed evidence of deep invasion in the absence of superficial involvement, suggesting possible hematogenous spread. Tissue culture of $C$. parapsilosis, an organism that had previously been cultured from the patient's blood and peritoneal fluid, also suggested dissemination from a distant site. Seeding of the skin from internal sources of infection, such as was seen in this case, is not infrequent [4].

Histopathology and culture are essential for a definitive diagnosis of mucormycosis. A multifaceted approach should be undertaken for management, including systemic antifungal treatment, prompt and aggressive surgical debridement, and reversal of underlying risks and contributing comorbidities whenever possible [5-7]. High-dose liposomal or lipid-complex amphotericin is the preferred monotherapy for mucormycosis $[3,8]$ and renal function should be monitored due to its nephrotoxicity. Other regimens include combination therapies of lipid-based amphotericin with an echinocandin and/or an azole, generally itraconazole or posaconazole $[3,8]$.

Adjunct treatments such as hyperbaric oxygen have also demonstrated some benefit in case reports $[3,8]$. Other treatment options that have not been well-studied include iron chelation and adjunctive cytokine therapy with interferon- $\gamma$ or granulocyte-macrophage colony-stimulating factor to promote phagocytic activity [3]. Surgical debridement of localized disease is essential and enhances cure rates by preventing dissemination and managing necrosis that may not be adequately addressed by systemic antifungals [3, 7]. Prompt and aggressive surgical debridement and adjunctive antifungal therapy of localized cutaneous mucormycosis have been shown to be associated with a mortality rate of $<10 \%$ [3].

This case illustrates the need for maintaining a broad differential diagnosis for a necrotic ulcer in an immunocompromised host. Skin histopathology and tissue culture are both essential in spite of coincident broad antimicrobial coverage, especially in a vulnerable, immunocompromised population. Prompt and aggressive treatment of the patient with cutaneous mucormycosis is imperative, as demonstrated in this case.

\section{Statement of Ethics}

The manuscript was prepared in compliance with all ethics and confidentiality guidelines and principles.

\section{Disclosure Statement}

The authors have no conflicts of interest to disclose. 
Yu et al:: Congenital Tricuspid Atresia with a New Axillary Lesion after an Orthotopic Heart Transplant

\section{References}

1 Petrikkos G, Skiada A, Lortholary O, Roilides E, Walsh TJ, Kontoyiannis DP. Epidemiology and clinical manifestations of mucormycosis. Clin Infect Dis. 2012 Feb;54 Suppl 1:S23-34.

2 Vulsteke JB, Deeren D. Cutaneous mucormycosis. Transpl Infect Dis. 2019 Apr;21(2):e13039.

3 Spellberg B, Edwards J Jr, Ibrahim A. Novel perspectives on mucormycosis: pathophysiology, presentation, and management. Clin Microbiol Rev. 2005 Jul;18(3):556-69.

4 Roden MM, Zaoutis TE, Buchanan WL, Knudsen TA, Sarkisova TA, Schaufele RL, et al. Epidemiology and outcome of zygomycosis: a review of 929 reported cases. Clin Infect Dis. 2005 Sep;41(5):634-53.

5 Castrejón-Pérez AD, Welsh EC, Miranda I, Ocampo-Candiani J, Welsh O. Cutaneous mucormycosis. An Bras Dermatol. 2017 May-Jun;92(3):304-11.

6 Skiada A, Petrikkos G. Cutaneous mucormycosis. Skinmed. 2013 May-Jun;11(3):155-9.

7 Suthananthan AE, Koek SA, Sieunarine K. Cutaneous mucormycosis in an immunocompromised patient: a case report. J Surg Case Rep. 2017 Mar;2017(3):rjx056.

8 Cornely OA, Arikan-Akdagli S, Dannaoui E, Groll AH, Lagrou K, Chakrabarti A, et al.; European Society of Clinical Microbiology and Infectious Diseases Fungal Infection Study Group; European Confederation of Medical Mycology. ESCMID and ECMM joint clinical guidelines for the diagnosis and management of mucormycosis 2013. Clin Microbiol Infect. 2014 Apr;20 Suppl 3:5-26. 